\title{
Series: Cardiovascular outcome trials for diabetes drugs Liraglutide and LEADER
}

\author{
MILES FISHER
}

\begin{abstract}
LEADER (Liraglutide Effect and Action in Diabetes: Evaluation of Cardiovascular Outcome Results) was an FDA-mandated cardiovascular outcome trial with liraglutide and was the first trial with a glucagon-like peptide-1 (GLP-1) receptor agonist to demonstrate a significant reduction in cardiovascular events. It compared liraglutide and placebo in 9,340 people with type 2 diabetes and either existing cardiovascular disease or age $>60$ years with at least one cardiovascular risk factor. LEADER demonstrated superiority for major cardiovascular events (cardiovascular death, non-fatal myocardial infarction, non-fatal stroke), and cardiovascular deaths were significantly reduced, as was all-cause mortality. Hospitalisation for heart failure, which was a secondary outcome, was not significantly reduced. Compared with the EMPA-REG OUTCOME trial, the curves for major adverse cardiovascular events in LEADER separated later, and the absence of a clear effect on hospitalisation for heart failure or on estimated glomerular filtration rate suggests that the mechanism of cardiovascular benefit for liraglutide was different from that for empagliflozin

Br J Diabetes 2020:20:142-144
\end{abstract}

Key words: diabetes, cardiovascular outcome trial, liraglutide

\section{Introduction}

Licensing requirements for new anti-diabetes drugs changed in the USA in 2008, and between 2008 and 2020 a dedicated randomised controlled cardiovascular outcome trial (CVOT) was usually required either before or after licensing ${ }^{1,2}$ This series describes and summarises the results of these CVOTs in the order in which they were published, describing the primary endpoint and important secondary outcomes from the principal publication, and directs attention to important subsequent publications of data from subgroups and post hoc analyses. LEADER (Liraglutide Effect and Action in Diabetes: Evaluation of Cardiovascular Outcome Results)

Address for correspondence: Professor Miles Fisher Department of Diabetes, Endocrinology \& Clinical Pharmacology, Glasgow Royal Infirmary, 84 Castle Street, Glasgow G4 OSF, UK E-mail: miles.fisher@ggc.scot.nhs.uk

https://doi.org/10.15277/bjd.2020.267 was the second published FDA-mandated CVOT using a glucagonlike peptide-1 (GLP-1) receptor agonist. ${ }^{3}$ It came after three negative trials with dipeptidyl peptidase 4 (DPP-4) inhibitors ${ }^{4-6}$ and a negative trial with the GLP-1 receptor agonist lixisenatide, ${ }^{7}$ whereas the EMPA-REG OUTCOME trial with empagliflozin had demonstrated reductions in major adverse cardiovascular events (MACE), cardiovascular deaths, all-cause mortality and hospitalisation for heart failure. ${ }^{8}$

\section{Background}

Liraglutide was approved in 2009 by the EMA for use in Europe and in 2010 by the FDA for use in the USA. It was the second GLP1 receptor agonist to be approved by the FDA and EMA following twice-daily exenatide, which was approved before the new licensing requirements. The cardiovascular safety of liraglutide was assessed in a patient-level pooled analysis of 15 phase 2 and phase 3 clinical development studies. ${ }^{9}$ Major adverse cardiovascular events (MACE) were identified by querying the study database using Medica Dictionary for Regulatory Activities (MeDRA) terms combined with serious adverse events recorded by study investigators. The incidence ratio for MACE associated with liraglutide was 0.73 (95\% $\mathrm{Cl} 0.38$ to 1.41 ) versus all comparator drugs, satisfying the FDA safety criteria requirements pre-licensing.

\section{LEADER}

A paper describing the design and baseline characteristics of LEADER was published in 2013. ${ }^{10}$ The principal LEADER results were presented in 2016 at the meeting of the American Diabetes Association and published simultaneously in the New England Journal of Medicine. ${ }^{3}$ The design of the study and key baseline characteristics of LEADER are shown in Box 1.

In LEADER there was a significant reduction in MACE with liraglutide, demonstrating superiority (Figure 1, Box 2). In the liraglutide group there were statistically significant reductions in cardiovascular death and death from any cause. There were no significant differences in the rates of myocardial infarction, stroke or hospitalisation for heart failure. The rate of gastrointestinal events was increased with liraglutide and there was no increase in other adverse events. There were significantly fewer episodes of severe hypoglycaemia and confirmed hypoglycaemia in the liraglutide group compared with the placebo group. There were 13 patients with pancreatic cancer in the liraglutide group and 5 in the placebo group. Acute pancreatitis occurred in 18 patients in the liraglutide group and 23 in the placebo group. 


\section{Box 1 Key features of LEADER ${ }^{3,10}$}

- LEADER compared liraglutide (1.8 $\mathrm{mg}$ or the maximum tolerated dose) versus placebo for a median follow-up of 3.8 years in 9,340 subjects

- Mean age of subjects was 64 years with a mean duration of diabetes of 13 years

- Mean baseline $\mathrm{HbA}_{1 \mathrm{c}}$ was $8.7 \%(72 \mathrm{mmol} / \mathrm{mol})$

- $81 \%$ of subjects had established atherosclerotic disease, $31 \%$ prior myocardial infarction, $39 \%$ prior revascularisation, $16 \%$ prior stroke or transient ischaemic attack and 14\% investigator-reported heart failure, but this diagnosis was not well characterised

- $76 \%$ of subjects were on metformin, $51 \%$ sulfonylureas, $6 \%$ thiazolidinediones, $44 \%$ insulin

Figure 1. 3.8-year event rates (\%) comparing liraglutide and placebo for major adverse cardiovascular events (MACE), total mortality, cardiovascular mortality, non-fatal myocardial infarction, non-fatal stroke and hospitalisation for heart failure (HFH)

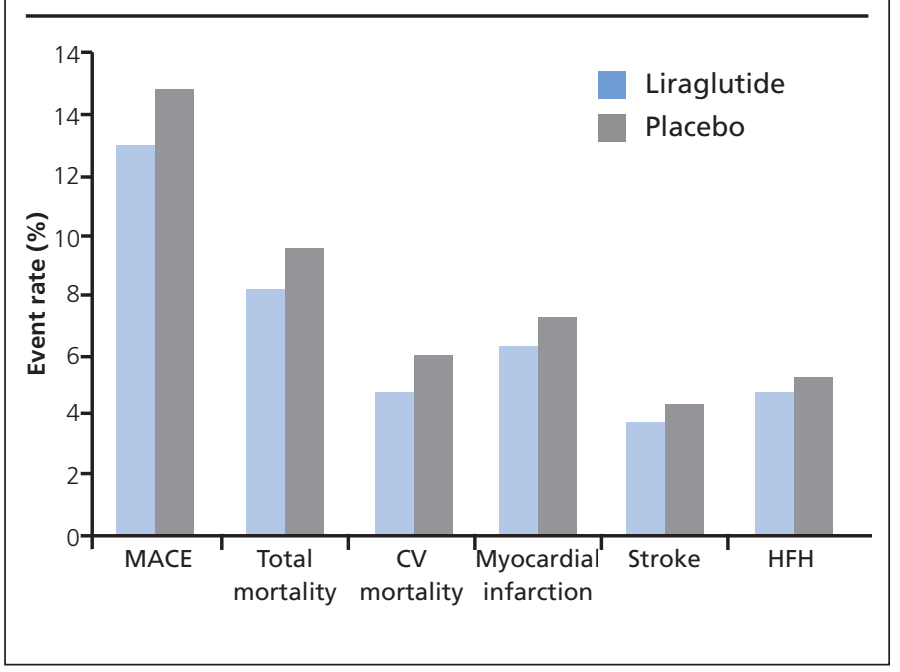

\section{Other results from LEADER}

Further publications from LEADER are shown in Box 2. A detailed publication on pre-specified renal outcomes was published one year later in the New England Journal of Medicine. ${ }^{11}$ The pre-specified secondary renal outcome was a composite of new-onset persistent macroalbuminuria, persistent doubling of the serum creatinine level, end-stage renal disease or death due to renal disease, and it was significantly reduced with liraglutide. The result was driven by reductions in new onset of persistent macroalbuminuria, and there was no difference in persistent doubling of serum creatinine or the need for renal replacement therapy.

\section{Discussion}

LEADER was the first CVOT with a GLP-1 receptor agonist to demonstrate significant cardiovascular benefits with a reduction in MACE. The pattern of cardiovascular benefit differed from that seen in EMPA-REG OUTCOME. ${ }^{8}$ In both trials there was a significant reduction in cardiovascular deaths and all-cause mortality, but in
Box 2 Results of the LEADER trial

Principal result

- Significant reduction in MACE, cardiovascular death, and death from any cause ${ }^{3}$

\section{Other results from LEADER}

- A pre-specified composite renal outcome was reduced in the liraglutide group, driven primarily by reductions in new onset of persistent macroalbuminuria ${ }^{11}$

- MACE and all-cause mortality were significantly reduced by liraglutide in patients with an estimated glomerular rate $<60 \mathrm{~mL} / \mathrm{min} / 1.73 \mathrm{~m}^{2} 13$

- Patients in the liraglutide group had lower $\mathrm{HbA}_{1 c}$, with an estimated reduction at 36 months of $0.4 \%$, with a lower risk of hypoglycaemia, including severe hypoglycaemia, and less glycaemic deterioration ${ }^{14,15}$

- When both groups were combined, patients with severe hypoglycaemia had longer duration of diabetes, more use of baseline insulin, and were more likely to experience a MACE, with higher risk shortly after hypoglycaemia ${ }^{15}$

- Overall there were similar proportions of diabetes-related foot ulcers in the two groups, but there was a significant reduction in amputations with liraglutide versus placebo ${ }^{16}$

- Patients in the liraglutide group reported a modest but significant benefit in patient-reported health status using the European Quality of life Questionnaire (EQ-50) compared with placebo ${ }^{17}$

- There was a significantly increased risk of acute gall bladder or biliary disease with liraglutide versus placebo (141 events versus 88 events $)^{18}$

- There were numerically fewer events of acute pancreatitis; amylase and lipase levels increased in the liraglutide group but did not predict the future risk of acute pancreatitis ${ }^{19}$

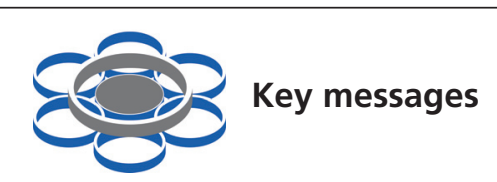

- LEADER was the sixth published cardiovascular outcome trial of a new diabetes drug, the second with a GLP-1 receptor agonist, comparing liraglutide and placebo

- In LEADER, liraglutide significantly reduced major adverse cardiovascular events, cardiovascular death and all-cause mortality, with no effect on hospitalisation for heart failure

- Subsequent cardiovascular outcome trials with GLP-1 receptor agonists have demonstrated variable results

- The possible mechanisms of benefit of liraglutide and other GLP-1 receptor agonists are under investigation

LEADER the benefit emerged later than in EMPA-REG OUTCOME and no reduction was observed in heart failure events. Similarly, the pattern of renal benefit differed from the detailed renal results of EMPA-REG OUTCOME, ${ }^{12}$ with no effect on doubling of serum creatinine. The LEADER investigators suggested that the cardiovascular benefits in LEADER might be related to modified progression of atherosclerotic vascular disease. 
Conflict of interest The author has received personal fees from AstraZeneca, Boehringer Ingelheim, Eli Lilly, Lexicon, MSD, NAPP, Novo Nordisk and Sanofi, outside the submitted work.

\section{Funding None.}

\section{References}

1. Food and Drug Administration. Guidance for industry. Diabetes mellitus - evaluating cardiovascular risk in new antidiabetic therapies to treat type 2 diabetes. Silver Spring, MD: US Department of Health and Human Services, Food and Drug Administration, Center for Drug Evaluation and Research (CDER), 2008. Available from: https://www.fda.gov/regulatory-information/search-fda-guidance-documents/diabetes-mellitus-evaluating-cardiovascular-risk-new-antidiabetic-therapies-treat-type-2-diabetes (accessed 1 March 2020).

2. Chong WH, Yanoff LB, Andraca-Carrera E, Thanh Hai M. Assessing the safety of glucose-lowering drugs - a new focus for the FDA. $N$ Engl J Med 2020;383:1199-202. https://doi.org/10.1056/NEJMp2004889

3. Marso SP, Daniels GH, Brown-Frandsen $K$, et al, for the LEADER Steering Committee on behalf of the LEADER Trial Investigators. N Engl J Med 2016;375:311-22. https://doi.org/10.1056/NEJMoa1603827

4. Scirica BM, Bhatt DL, Braunwald E, et al, for the SAVOR-TIMI 53 Steering Committee and Investigators. Saxagliptin and cardiovascular outcomes in patients with type 2 diabetes mellitus. N Eng/ J Med 2013;369:131726. https://doi.org/10.1056/NEJMoa1307684

5. White WB, Cannon CP, Heller SR, et al, for the EXAMINE Investigators. Alogliptin after acute coronary syndrome in patients with type 2 diabetes. N Engl J Med 2013;369:1327-35. https://doi.org/10.1056/NEJMoa1305889

6. Green JB, Bethel A, Armstrong PW, et al, for the TECOS Study Group. Effect of sitagliptin on cardiovascular outcomes in type 2 diabetes. N Eng/ J Med 2015;373:232-42. https://doi.org/10.1056/NEJMoa1501352

7. Pfeffer MA, Claggett $B$, Diaz R, et al, for the ELIXA Investigators. Lixisenatide in patients with type 2 diabetes and acute coronary syndrome. N Engl J Med 2015;373:2247-57. https://doi.org/10.1056/NEJMoa1509225

8. Zinman B, Wanner C, Lachin JM, et al, for the EMPA-REG OUTCOME Investigators. Empagliflozin, cardiovascular outcomes, and mortality in type 2 diabetes. N Engl J Med 2015;373:2117-28. https://doi.org/ 10.1056/NEJMoa1504720

9. Marso SP, Lindsey JB, Stolker JM, et al. Cardiovascular safety of liraglutide assessed in a patient-level pooled analysis of phase 2-3 liraglutide clinical development studies. Diabetes Vasc Dis Res 2011;8:237-40. https://doi.org/10.1177/1479164111408937

10. Marso SP, Poulter NR, Nissen SE, et al. Design of the Liraglutide Effect and Action in Diabetes: Evaluation of Cardiovascular Outcome Results
(LEADER) trial. Am Heart J 2013;166:823-30. https://doi.org/10.1016/ j.ahj.2013.07.012

11. Mann JFE, Orsted DD, Brown-Frandsen $K$, et al, for the LEADER Steering Committee and Investigators. Liraglutide and renal outcomes in type 2 diabetes. N Eng/ J Med 2017;377:839-48. https://doi.org/10.1056/NEJMoa1616011

12. Wanner C, Inzucchi SE, Lachin JM, et al, for the EMPA-REG OUTCOME Investigators. Empagliflozin and progression of kidney disease in type 2 diabetes. N Engl J Med 2016;375:323-34. https://doi.org/10.1056/NEJMoa1515920

13. Mann JFE, Fonseva V, Mosensen O, et al. The LEADER Publication Committee on behalf of the LEADER Trial Investigators. Effects of liraglutide versus placebo on cardiovascular events in patients with type 2 diabetes mellitus and chronic kidney disease results from the LEADER trial. Circulation 2018;138:2908-18. https://doi.org/10.1161/CIRCULATIONAHA.118.036418

14. Zinman B, Nauck MA, Bosch-Traberg $\mathrm{H}$, et al. The LEADER Publication Committee on behalf of the LEADER Trial Investigators. Liraglutide and glycaemic outcomes in the LEADER trial. Diabetes Ther 2018;9:238392. https://doi.org/10.1007/s13300-018-0524-z

15. Zinman $B$, Marso SP, Christiansen $E$, et al, and the LEADER Publication Committee on behalf of the LEADER Trial Investigators. Diabetes Care 2018:41:1783-91. https://doi.org/10.2337/dc17-2677

16. Dhatariya K, Bain SC, Buse JB, et al, and the LEADER Publication Committee on behalf of the LEADER Trial Investigators. The impact of liraglutide on diabetes-related foot ulceration and associated complications in patients with type 2 diabetes at high risk for cardiovascular events: results from the LEADER trial. Diabetes Care 2018;41:2229-35. https://doi.org/10.2337/dc18-1094

17. Nauck MA, Buse JB, Mann JFE, et al, and the LEADER Publication Committee on behalf of the LEADER Trial Investigators. Health-related quality of life in people with type 2 diabetes participating in the LEADER trial. Diabetes Obes Metab 2019;21:525-32. https://doi.org/10.1111/ dom.13547

18. Nauck MA, Muus Ghorbani ML, Kreiner E, et al, and the LEADER Publication Committee on behalf of the LEADER Trial Investigators. Effects of liraglutide compared with placebo on events of acute gallbladder or biliary disease in patients with type 2 diabetes at high risk for cardiovascular events in the LEADER randomized trial. Diabetes Care 2019; 42:1912-20. https://doi.org/10.2337/dc19-0415

19. Steinberg W, Buse JB, Ghorbani MLM, et al, and the LEADER Steering Committee on behalf of the LEADER Trial Investigators. Amylase, lipase, and acute pancreatitis in people with type 2 diabetes treated with liraglutide: results from the LEADER randomized trial. Diabetes Care 2017;40:966-72. https://doi.org/10.2337/dc16-2747 